\title{
Neurosurgery clinical registry data collection utilizing Informatics for Integrating Biology and the Bedside and electronic health records at the University of Rochester
}

\author{
Christine A. Pittman, MPH, and Amrendra S. Miranpuri, MD \\ Department of Neurosurgery, University of Rochester, New York
}

In a population health-driven health care system, data collection through the use of clinical registries is becoming imperative to continue to drive effective and efficient patient care. Clinical registries rely on a department's ability to collect high-quality and accurate data. Currently, however, data are collected manually with a high risk for error. The University of Rochester's Department of Neurosurgery in conjunction with the university's Clinical and Translational Science Institute has implemented the integrated use of the Informatics for Integrating Biology and the Bedside (i2b2) informatics framework with the Research Electronic Data Capture (REDCap) databases.

http://thejns.org/doi/abs/10.3171/2015.9.FOCUS15382

KEY WORDS i2b2; clinical outcomes; database design

$\mathrm{T}$ HERE is an expanded need for integrating evidencebased medicine into neurosurgical practices. However, the 2 main sources of data-administrative data sets and clinical registries - have their unique pitfalls. In a health care system increasingly informed by evidence-based medicine, both types of data sets are being used to study the outcomes and effectiveness of services provided. ${ }^{1}$ Conversely, many of the administrative databases are unable to provide detailed patient-level data that impact outcomes analysis. ${ }^{3}$ Therefore, we rely heavily on clinical registries to measure outcomes. Currently, the success of data collection and management depends on trained personnel to manually enter data into a database and an extensive infrastructure to maintain the data. Unfortunately, hospitals are limited by the financial burden of needing personnel to manually enter data.

In this paper we describe the University of Rochester's unique data collection method and its ability to integrate the use of Informatics for Integrating Biology and the Bedside (i2b2) with Research Electronic Data Capture (REDCap) databases to successfully capture data with little input from a data extractor.

\section{Role of i2b2 in Collecting Clinical Data}

i2b2 is an open-source informatics framework developed by the National Center for Biomedical Computing based at Partners HealthCare System to enable researchers to query clinical data from multiple clinical systems for research purposes. i2b2 utilizes a hive system, which maintains server-side software modules that either store data or contain analysis methods that facilitate the use of data. ${ }^{2}$ At the University of Rochester, we use an Oracle platform to integrate a data repository and the i2b2 software. Fulltime employees (FTEs) are required to manage the $\mathrm{i} 2 \mathrm{~b} 2$ platform and continue to develop the tool. Currently, we have 2 project coordinators and 3 programmers to build and maintain the software. $\mathrm{i} 2 \mathrm{~b} 2$ is accessible to all departments within the medical center and is used by more than 16 departments. Over the past 2 years, the university has spent time improving the data extraction mechanism and continues to improve user feasibility through the use of open forums to better match the needs of clinical researchers across the university. Our Clinical and Translational Science Institute has estimated that an i2b2 project takes,

ABBREVIATIONS API = application programming interface; DOB = date of birth; EHR = electronic health record; FTE = full-time employee; i2b2 = Informatics for Integrating Biology and the Bedside; MRN = medical record number; N²OD = National Neurosurgery Quality and Outcomes Database; PRO = patient-reported outcome; REDCap $=$ Research Electronic Data Capture. 
on average, 15\% of an FTE's work week for its creation and set up and an additional 5\% for maintenance and error proofing of the database. However, as we continue to improve upon the use of the software, these time allocations are decreasing.

At the University of Rochester, we have linked data from our electronic health record (EHR), SoftLab (laboratory data), and Flowcast (our billing and scheduling process) systems for our 3 affiliated hospitals. Data from each system is copied into a data repository on a weekly basis. The data repository is an independent data entity with a complete copy of discrete EHR variables. Therefore, researchers and physicians are able to manipulate data as they please.

i2b2 has a graphical interface for 2 general uses. The first is to browse through the data repository to identify sets of patients that would be of interest for future projects such as volunteer subject recruitment, to support study feasibility, or to identify cohorts. The second use is to conduct an in-depth study on a specific population in support of outcome studies. ${ }^{2}$ Some examples of data currently available include patient demographics, diagnoses, medications, procedures, providers, laboratory results, microbiology results, patient questionnaires, and social histories. De-identified data are available for study feasibility assessment and planning. With proper institutional review board approval, users can request identified data for a user-specific $\mathrm{i} 2 \mathrm{~b} 2$ project, which can be exported for analysis.

When the University of Rochester implemented an EHR system in 2012, it became difficult for physicians and researchers to query data. The EHR system is useful for identifying individual patient-level data; however, a limited number of processes are available to generate reports and queries on a cohort of patients. ${ }^{2}$ Therefore, the University of Rochester developed a complementary tool to automate the process of moving clinical information from $\mathrm{i} 2 \mathrm{~b} 2$ to REDCap by using an innovative graphical user interface. REDCap, developed at Vanderbilt University and made freely available through the REDCap Consortium, is used for the collection and management of data for biomedical research. It allows collection of data through surveys and electronic case report forms and includes features to facilitate the collection of cross-sectional and longitudinal data. At the University of Rochester, we can use the tool to map clinical data in $\mathrm{i} 2 \mathrm{~b} 2$ to specific fields in a REDCap project by using an application programming interface (API). Figure 1 illustrates the process of gathering data using $\mathrm{i} 2 \mathrm{~b} 2$ and then exporting data to REDCap.

The use of $\mathrm{i} 2 \mathrm{~b} 2$ at an institutional level provides an institution with the ability to conduct quality projects and potential clinical research initiatives in a more costeffective manner. Currently, clinical researchers spend hours mining through the EHR system for information or even conducting telephone screenings to better identify potential patients for a clinical research study. i $2 \mathrm{~b} 2$ was designed as a clinical query tool and later developed into a database creation tool. Figure 2 illustrates the percentage of an FTE's time required for the creation and maintenance of a clinical registry using this method.

\section{University of Rochester's Approach to Collecting Clinical Data}

In preparation for joining national clinical registries, we created a comprehensive database that analyzed patient outcomes after treatment for a degenerative spine diagnosis (including ICD-9 codes 720.0-724.9). Using the Department of Neurosurgery's Evaluation and Management report from 2009 to 2014, we identified 6050 adults (age > 18 years) who had been evaluated for a degenerative spine diagnosis. A patient list was created in Microsoft Excel including the patient's first and last name and medical record number (MRN). Patient lists needed to include date of enrollment and 1 of the following options when uploading: 1) site (hospital name), patient's first and last name, and patient's date of birth (DOB); 2) MRN and DOB; or 3) patient's first and last name and MRN. The date of enrollment for all patients in this study was their date of initial evaluation by a neurosurgeon at the University of Rochester. These combinations of data allowed $\mathrm{i} 2 \mathrm{~b} 2$ to sync the patient information with information found in the data repository.

When creating our REDCap database we included variables such as patient demographics, medications, pain score, procedures such as injection infusions and/or surgery, and blood loss. The REDCap database was linked to our i2b2 database through an API (Fig. 1). Using the variable list in $\mathrm{i} 2 \mathrm{~b} 2$, we mapped the desired variables to the corresponding fields in the REDCap project (Fig. 3). By syncing the variables with REDCap, the information from the data repository was sent directly to the REDCap database.

This approach led to the collection of, on average, $80 \%$ of the basic variables for national registries such as the National Neurosurgery Quality and Outcomes Database ( $\left.\mathrm{N}^{2} \mathrm{QOD}\right)$. However, the $20 \%$ of variables not collected were a part of surgical notes, physician notes, and other nondiscrete fields. For example, of the 6050 patients included in our spine database, 5562 patients had a principal diagnosis code completed, which is $92 \%$ of the patient population (Table 1). Patient demographics such as name, DOB, address, insurance type, age, sex, race, and ethnicity were completed $100 \%$ of the time.

Given the nature of passive data entry, we found it imperative to choose a simple random sample of patients and conduct manual chart reviews. For the 200 patients selected for review, the data included in REDCap were $99 \%$ accurate. We found that, depending on the physician type, certain variables were included in different parts of the patient chart. Moreover, i2b2 relies on data "rows" to pull information, so height and weight are often found in various aspects of the $\mathrm{i} 2 \mathrm{~b} 2$ portal. These quality assurance checks allowed us to pinpoint the exact location of variables in the i2b2 portal. Once these variables are mapped, they will continue to be mapped every time a query is run and when new patients are added.

\section{Future Directions}

i2b2 is reliable for discrete variables; however, many of the national clinical registries require variables that are normally collected in a note or text format. Thus, there 

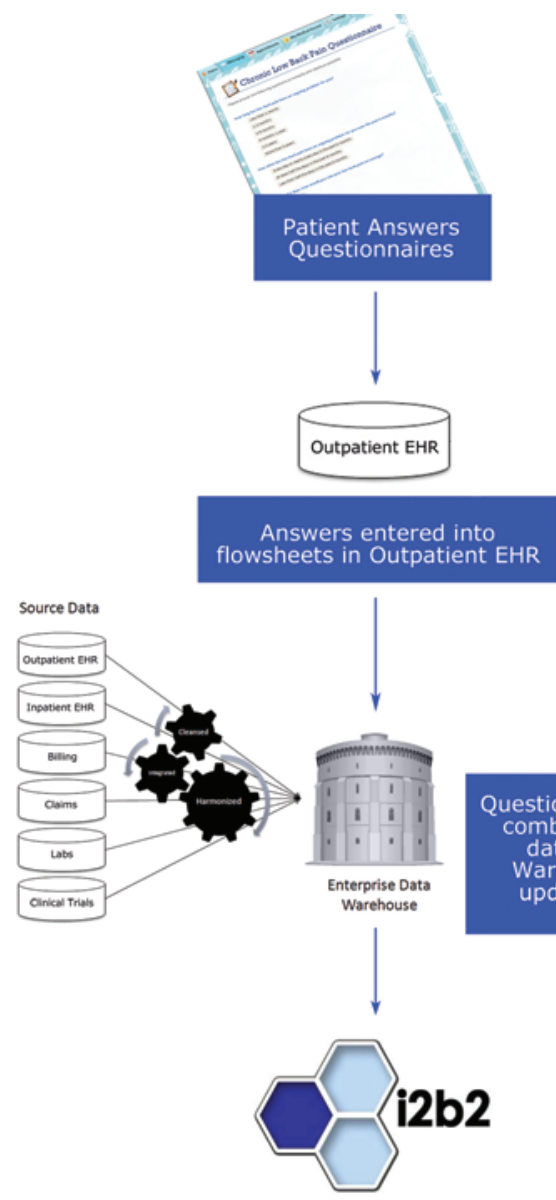

\section{REDCap}

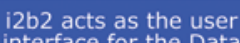

interface for the Data

Warehouse
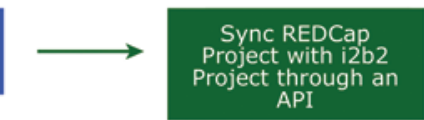

Create a REDCap

Database Project

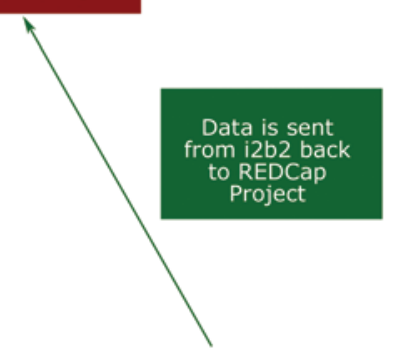

FIG. 1. Schematic showing University of Rochester i2b2 process, which features the i2b2/REDCap integration.

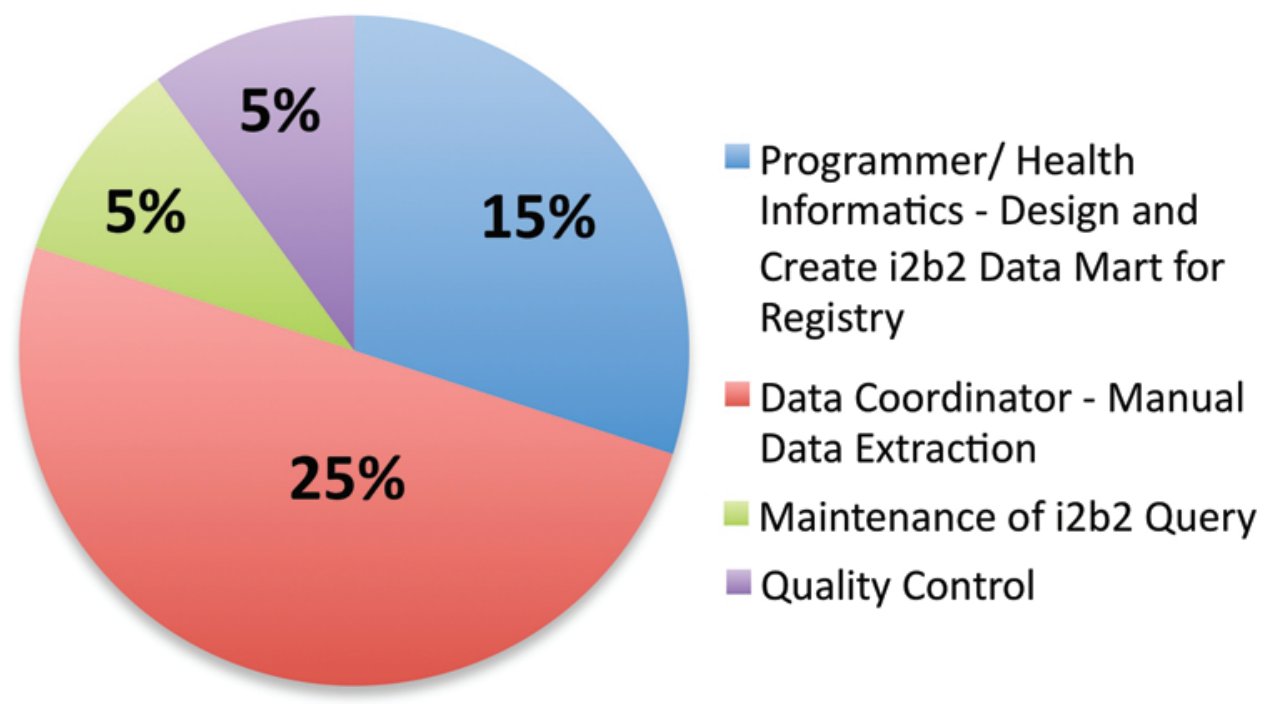

FIG. 2. Percentage of an FTE's time required to create and maintain a clinical registry using i2b2. Although the National Neurosurgery Quality and Outcomes Database standard requires $50 \%$ of an FTE's time per module for data collection, we found that only $15 \%$ of the data coordinator's role would be dedicated to creating the registry by using i2b2 and REDCap. Generally, the creation of the registry takes approximately 1 week. An additional $5 \%$ of the data coordinator's role would be required for maintenance of the database. Therefore, 1 half-time data coordinator (or $0.5 \mathrm{FTE}$ ) could be responsible for at least 2 modules. 


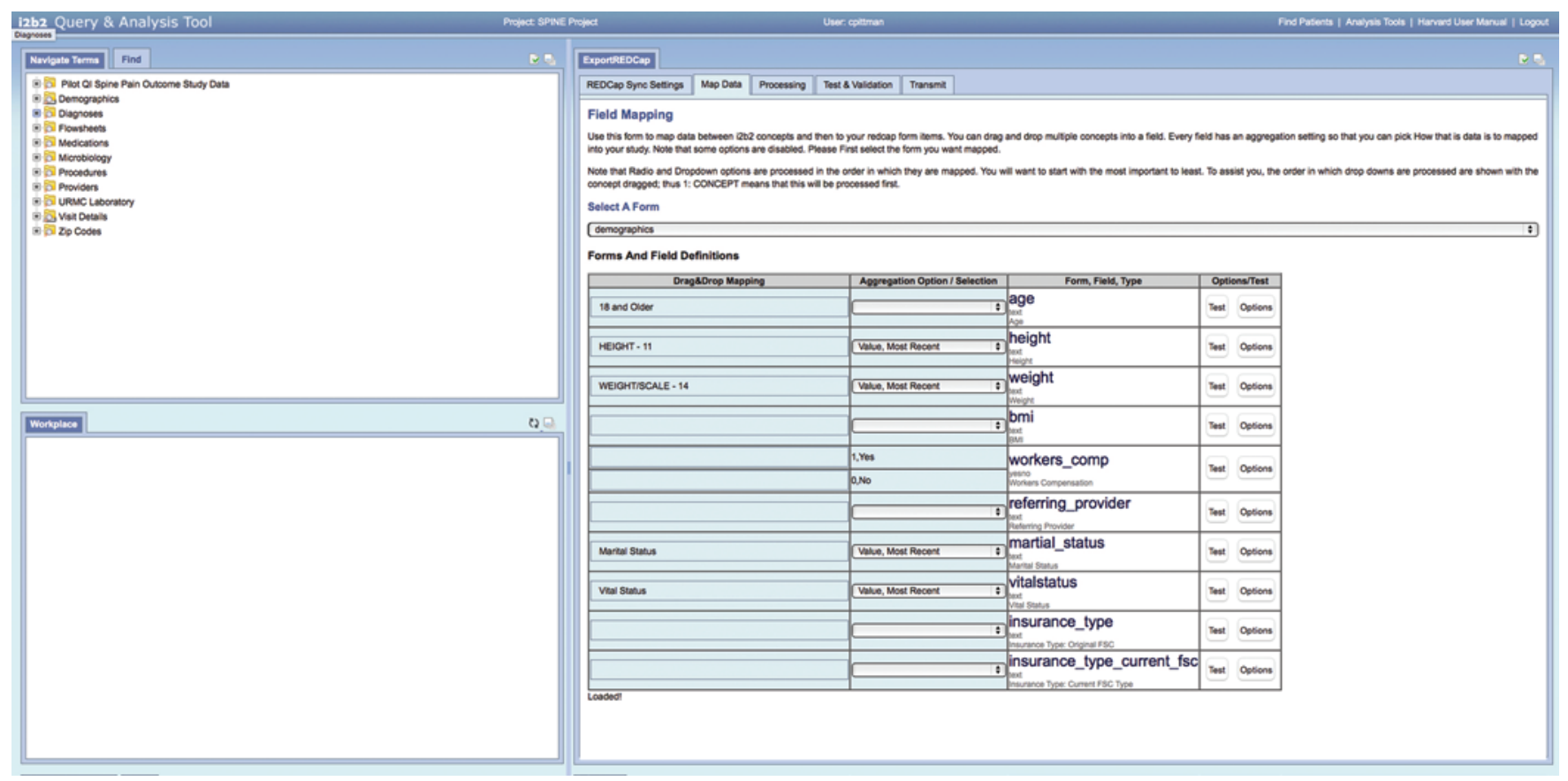

FIG. 3. Screenshot showing i2b2 to REDCap mapping. The navigation terms (far left column) are the i $2 \mathrm{~b} 2$ variables. These variables are mapped to the corresponding "Form, Field, Type" variable name found in REDCap (wide white column on far right). By dragging a navigation term to the "Drag\&Drop Mapping" section (first blue column on right), data from the data repository are linked to REDCap.

TABLE 1. Principal diagnosis codes included in the spine database

\begin{tabular}{lc}
\hline \multicolumn{1}{c}{ Diagnosis } & Percentage of Patients \\
\hline Thoracic disc displacement & $0.20 \%$ \\
\hline Cervical disc disorder w/ myelopathy & $0.25 \%$ \\
\hline Thoracic disc degeneration & $0.25 \%$ \\
\hline Disorder of coccyx, not elsewhere classified & $0.27 \%$ \\
\hline Other back symptoms & $0.31 \%$ \\
\hline Postlaminectomy syndrome NOS & $0.52 \%$ \\
\hline Pain in thoracic spine & $0.79 \%$ \\
\hline Backache NOS & $0.85 \%$ \\
\hline Postlaminectomy syndrome, cervical & $0.99 \%$ \\
\hline Sciatica & $1.33 \%$ \\
\hline Spondylolisthesis & $1.56 \%$ \\
\hline Lumbosacral neuritis NOS & $1.91 \%$ \\
\hline Cervical spinal stenosis & $2.46 \%$ \\
\hline Cervical spondylosis w/ myelopathy & $3.58 \%$ \\
\hline Postlaminectomy syndrome, lumbar & $3.97 \%$ \\
\hline Cervical disc degeneration & $4.12 \%$ \\
\hline Cervical disc displacement & $4.40 \%$ \\
\hline Cervicalgia & $6.90 \%$ \\
\hline Cervical spondylosis & $10.10 \%$ \\
\hline Lumbago & $11.36 \%$ \\
\hline Lumbosacral spondylosis & $12.01 \%$ \\
\hline Lumbar/lumbosacral disc degeneration & $13.79 \%$ \\
\hline Lumbar disc displacement & $16.95 \%$ \\
\hline Other (includes disc degeneration NOS) & $1.11 \%$ \\
\hline &
\end{tabular}

NOS $=$ not otherwise specified. are 2 approaches to dealing with such data. The first is the incorporation of natural language processing, and the second is the use of smart data elements in physician notes. We chose to customize our physician notes with smart data elements that allow physicians to simply click variables on the left side of the screen, which are then incorporated into the clinical note. Figure 4 shows the format of smart data elements that can simply be clicked and added into the physician notes. These variables are collected in flow sheets, which can then be exported into the data repository. Smart data elements can be customized for each provider; however, in an effort to standardize data collection to improve the quality and accuracy of data, we have standardized the smart data elements included at the end of the physician notes.

Clinical registries often include the use of patient-reported outcome (PRO) measures. Within our EHR system, we have built patient questionnaires that resemble the Oswestry Disability Index, STarT Back Tool, visual analog scale, and EQ-5D. ${ }^{4}$ The patients can answer these questionnaires through the MyChart patient portal, which collects the answers to the questionnaires in individual rows on a flow sheet. This will allow a more accurate collection of PRO data.

The integration of $\mathrm{i} 2 \mathrm{~b} 2$ and REDCap provides a reliable and effective option for accurately measuring and predicting clinical outcomes. As a university, we plan to enroll in the $\mathrm{N}^{2} \mathrm{QOD}$ for all the cervical, lumbar, and cerebrovascular modules. Because of its efficiency and ease of data export, i2b2 will allow us to collect data on all patients seen in the clinic for spine diseases and cerebrovascular disease. Rather than spending hours manually entering data into REDCap, data can be queried in $\mathrm{i} 2 \mathrm{~b} 2$ 


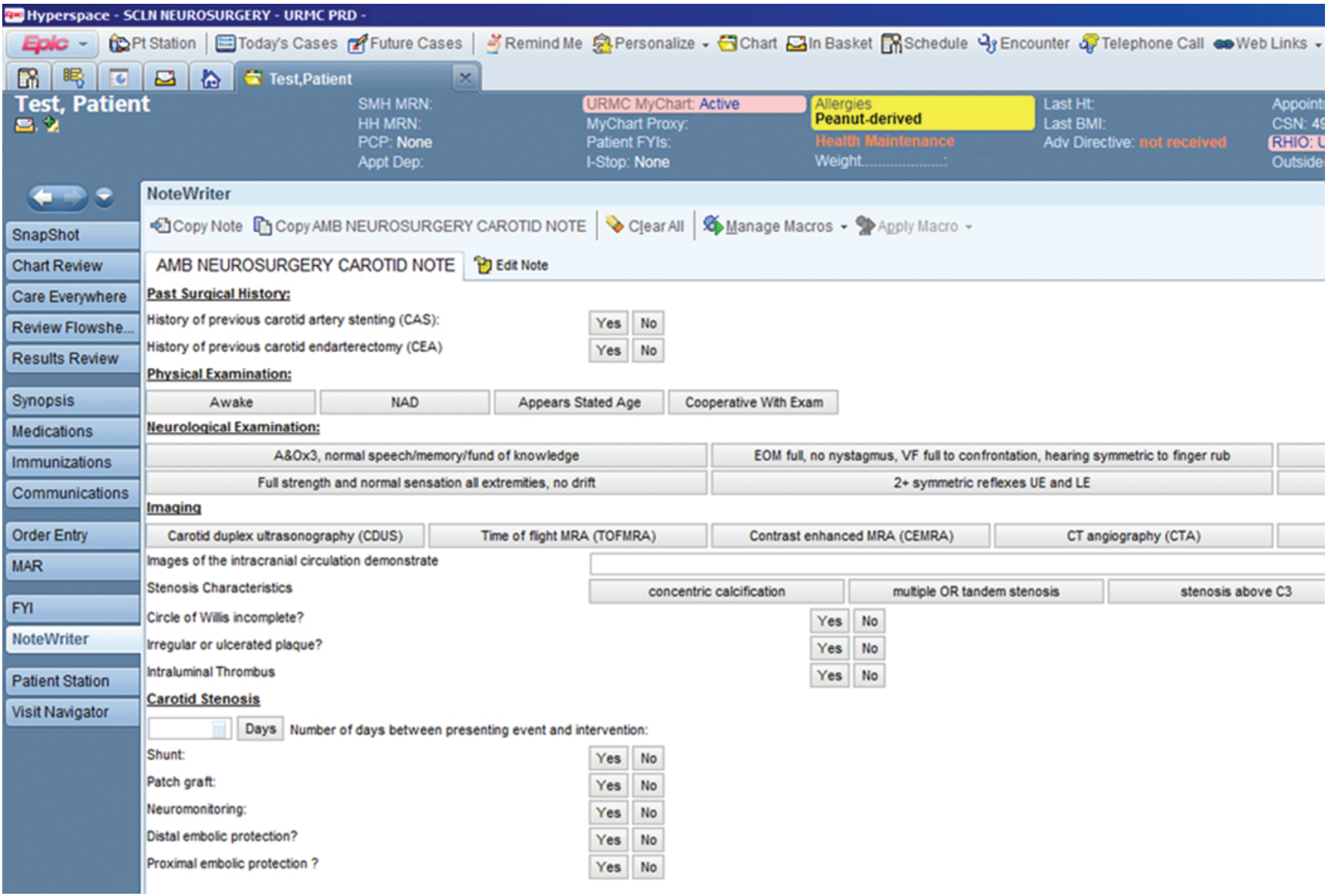

FIG. 4. Screenshot showing how the smart data elements look and function in the EHR. Simply clicking the variable will autopopulate the data into the note or text field. The information is recorded in a flowsheet row and relayed to the data repository on a weekly basis.

and pushed to REDCap in approximately 15 minutes. On a weekly basis, the database will be updated by adding additional patients to the "patient list." The data are then queried again and the REDCap database is updated. Because we are concerned about the quality of data collected, random samples of the patients will be reviewed and crosschecked with the EHR system to ensure accuracy. By incorporating the use of smart data elements in physician notes and PROs in the EHR patient portal, we aim to create an efficient means of collecting high-quality standardized data that can be used for analyses to come.

\section{Use of i2b2 for a Cerebrovascular Module}

We have designed operative notes, consultation notes, and follow-up clinical notes to contain smart data elements that will populate the i2b2 software. Although we recognize that every physician has a unique note style, smart elements can be used to complement the physician's notes and capture data more accurately than natural language processing. Patient health history, such as major surgery within the past year, history of comorbidities, smoking status, and so forth, can be pulled from the i2b2 through querying a patient's diagnoses list. However, some of this information is placed in the physician's initial consultation note. Therefore, creating smart data elements within the initial consultation will maintain the same information previously found in the physician notes while making the information a usable and extractable field. Procedurespecific information such as type of procedure performed, place of discharge, and number of days in the intensive care unit are fields that are already extractable in $\mathrm{i} 2 \mathrm{~b} 2$.

Other modules of the $\mathrm{N}^{2} \mathrm{QOD}$, such as the lumbar spine module, require operative details, such as the interbody graft used, which can also be incorporated into smart note elements. Although we hope that $\mathrm{i} 2 \mathrm{~b} 2$ or passive data entry mechanisms like $\mathrm{i} 2 \mathrm{~b} 2$ can eliminate the need for full-time data extractors, we recognize that there is a limit. In the lumbar spine module, a data extractor will have to collect data regarding the manner in which the interbody graft was placed. However, even though there is still a small need for a data extractor, the ability of i $2 \mathrm{~b} 2$ to collect more data in a cost-effective manner makes passive data entry an attractive alternative. Table 2 represents the difference between participating in $\mathrm{N}^{2} \mathrm{QOD}$ status quo and the potential differences $\mathrm{i} 2 \mathrm{~b} 2$ can provide to obviate the need for manual data extraction.

\section{Conclusions}

The unique aspect of i2b2 integrated with REDCap applies to a wide range of stakeholders. $i 2 b 2$ is an open- 
TABLE 2. Three-year cost effectiveness of $\mathrm{i} 2 \mathrm{~b} 2$

\begin{tabular}{|c|c|c|}
\hline Variable & $\mathrm{N}^{2} \mathrm{QOD}$ Status Quo & $\mathrm{N}^{2} \mathrm{QOD}$ w/ i2b2 \\
\hline \multirow[t]{2}{*}{ Cost of software } & $\mathrm{N}^{2} \mathrm{QOD}: \$ 13,000$ per yr & $\mathrm{N}^{2} \mathrm{QOD}: \$ 13,000$ per yr \\
\hline & i2b2: $\$ 0$ & i2b2: $\$ 0$ \\
\hline \multirow[t]{2}{*}{$\begin{array}{l}\text { Cost of data } \\
\text { coordinator }\end{array}$} & $\begin{array}{l}\text { 0.5 FTE per module: } \\
\$ 23,922^{*}\end{array}$ & $\begin{array}{l}1 \text { FTE for } 3 \text { modules: } \\
\$ 47,845^{*}\end{array}$ \\
\hline & $5 \%$ of programmer: $\$ 0$ & $\begin{array}{l}5 \% \text { of programmer: } \\
\$ 3052.50^{*}\end{array}$ \\
\hline $\begin{array}{l}\text { Amount of data } \\
\text { collected }\end{array}$ & $6-10$ patients a wk & All patients \\
\hline $\begin{array}{l}\text { Total cost for } 3 \\
\text { modules }\end{array}$ & $\$ 84,762$ & $\$ 63,897$ \\
\hline
\end{tabular}

* The salaries for a data coordinator and health care informatics programmer were based on the average salaries posted by the following sources: institutional human resources recommendations, glassdoor.com, and payscale.com. Although we recognize that salaries vary based on type of organization and job title, those listed represent our best estimates.

source program that can be incorporated into the EHR system and further customized to the specific institution. Our model for data collection can apply to various types of health care settings that will revolutionize the accuracy and quality of clinical registries.

\section{Acknowledgments}

We acknowledge Phillip Ng, MS, and Adam Tatro, MS, RN

\section{References}

1. Byrne MD, Jordan TR, Welle T: Comparison of manual versus automated data collection method for an evidence-based nursing practice study. Appl Clin Inform 4:61-74, 2013

2. Stey AM, Russell MM, Ko CY, Sacks GD, Dawes AJ, Gibbons MM: Clinical registries and quality measurement in surgery: a systematic review. Surgery 157:381-395, 2015

3. Theodosopoulos PV, Ringer AJ: Measuring outcomes for neurosurgical procedures. Neurosurg Clin N Am 26:265$269, \mathrm{x}, 2015$

4. Theodosopoulos PV, Ringer AJ, McPherson CM, Warnick RE, Kuntz C IV, Zuccarello M, et al: Measuring surgical outcomes in neurosurgery: implementation, analysis, and auditing a prospective series of more than 5000 procedures. J Neurosurg 117:947-954, 2012

\section{Disclosures}

The authors report no conflict of interest concerning the materials or methods used in this study or the findings specified in this paper.

\section{Author Contributions}

Conception and design: both authors. Acquisition of data: both authors. Analysis and interpretation of data: both authors. Drafting the article: both authors. Critically revising the article: both authors. Reviewed submitted version of manuscript: both authors. Approved the final version of the manuscript on behalf of both authors: Pittman. Administrative/technical/material support: Pittman. Study supervision: Miranpuri.

\section{Correspondence}

Christine A. Pittman, Department of Neurosurgery, University of Rochester, 601 Elmwood Ave., Box 670, Rochester, NY 14642. email: christine_pittman@urmc.rochester.edu. 